Proceedings of the Estonian Academy of Sciences,

2022, 71, 1, 3-15

https://doi.org/10.3176/proc.2022.1.01

Available online at www.eap.ee/proceedings

MATHEMATICS

\title{
Inverse problem to identify a space-dependent diffusivity coefficient in a generalized subdiffusion equation from final data
}

\author{
The paper is dedicated to the 100th birthday of Professor Ülo Lepik
}

Jaan Janno*, Kairi Kasemets and Nataliia Kinash

Department of Cybernetics, Tallinn University of Technology, Ehitajate tee 5, 19086 Tallinn, Estonia

Received 8 July 2021, accepted 11 October 2021, available online 31 January 2022

(C) 2022 Authors. This is an Open Access article distributed under the terms and conditions of the Creative Commons Attribution 4.0 International License CC BY 4.0 (http://creativecommons.org/licenses/by/4.0).

\begin{abstract}
An inverse problem to determine a space-dependent diffusivity coefficient in a one-dimensional generalized time fractional diffusion equation from final data is considered. The global uniqueness and local existence and stability of the solution to this problem is proved. Proof of these statements is based on the fixed-point principle and previously obtained results regarding an inverse source problem for a generalized subdiffusion equation.
\end{abstract}

Key words: inverse problem, final overdetermination, generalized fractional evolution equation.

\section{INTRODUCTION}

Equations with time fractional derivatives (containing power-type kernels) are used to model subdiffusion processes in self-similar media $[1,3,13]$. However, in many cases a medium under consideration is selfsimilar, which means that the character of the process changes when the time is rescaled. In such cases generalized fractional derivatives (containing more general kernels) are introduced to the equations [2,10, 20]. Generalized fractional derivatives involve more degrees of freedom and enable to better fit the models with real situations.

Often the medium parameters are a priori unknown and determined via solution to inverse problems that involve measurements of states of the processes. The states may be measured at final time moments. Final data are suitable for determination of space-dependent paramaters of the equations for two reasons: 1) unknown quantities and data are functions of the same type; 2) the resulting inverse problems are moderately ill-posed.

Problems to reconstruct space-dependent factors of source terms of subdiffusion equations containing usual or generalized fractional derivatives from final data have been studied in several papers $[8,9,11,14,18$, 21]. The analysis of such problems uses the Fourier expansion or positivity principles with the Fredholm alternative. A more general approach in a Hilbert space setting is presented in the recent paper [15].

Problems to recover reaction coefficents (potentials) from final data can be handled by means of the fixed-point principles on the basis of results obtained for inverse source problems [9]. Another approach

\footnotetext{
*Corresponding author, jaan.janno@ttu.ee
} 
to inverse coefficient problems for subdiffusion equations with usual fractional derivatives is based on Carleman estimates [17,23]. In case the interior of the domain is not accessible, boundary data can be used instead of the final measurements in order to recover space-dependent coefficients [7].

In the present paper we consider a problem to identify a space-dependent diffusivity coefficient in a one-dimensional subdiffusion equation containing a generalized time fractional derivative from the final data. We will prove the global uniqueness and local existence and stability for that problem. The analysis is based on previously obtained results regarding an inverse source problem for such an equation [9].

A surprising result is that the inverse diffusivity problem is less ill-posed than the corresponding inverse source problem: the solution to the former one depends continuously on the 1st derivative of the final measurement $u_{T}$ whereas the solution to the latter one depends continuously on the 2 nd derivative of $u_{T}$.

\section{PHYSICAL BACKGROUND AND FORMULATION OF INVERSE PROBLEM}

In the derivation of a differential equation to be considered in this paper we follow the approach presented in [16]. We assume the following constitutive relation with memory:

$$
Q(t, x)=-a(x) \frac{\partial}{\partial t} \int_{0}^{t} M(t-\tau) u_{x}(\tau, x) d \tau,
$$

where $t$ is the time, $x \in \mathbb{R}$ denotes a space variable, $Q$ represents the flux, $u$ is the state of the diffusion process, $M$ refers to a memory kernel and $a$ is the diffusivity. Plugging this relation into the conservation equation $u_{t}+Q_{x}=F$, where $F$ is the source function, we obtain the following generalized fractional diffusion equation:

$$
u_{t}(t, x)=\frac{\partial}{\partial t} M *\left(a(x) u_{x}(t, x)\right)_{x}+F(t, x),
$$

where $*$ denotes the time convolution, e.g. $z_{1} * z_{2}(t)=\int_{0}^{t} z_{1}(t-\tau) z_{2}(\tau) d \tau$.

Another method to derive the equation (2) is based on the continuous time random walk. Details can be found, e.g., in [2].

Suppose that there exists a time-dependent function $k$ such that $k * M(t) \equiv 1$. Then, applying the operator $k *$ to (2), we transform it to the following form that contains the explicit elliptic operator at the right-hand side:

$$
k * u_{t}(t, x)=\left(a(x) u_{x}(t, x)\right)_{x}+f(t, x), \quad f=k * F .
$$

The term $k * u_{t}(t, x)$ can be rewritten in a form that does not contain the first order time derivative of $u$ : $k * u_{t}(t, x)=\frac{\partial}{\partial t} k *[u(t, x)-u(0, x)]$. Thus, we obtain

$$
\frac{\partial}{\partial t} k *[u(t, x)-u(0, x)]=\left(a(x) u_{x}(t, x)\right)_{x}+f(t, x) .
$$

The operators $k * \frac{\partial}{\partial t}$ and $\frac{\partial}{\partial t} k *$ are the generalized fractional derivatives of Caputo and Riemann-Liouville type, respectively. We will use the following notation for the latter one:

$$
D_{t}^{\{k\}}=\frac{\partial}{\partial t} k * .
$$

In the usual fractional diffusion model, the kernels $M$ and $k$ are $M(t)=\frac{t^{\beta-1}}{\Gamma(\beta)}$ and $k(t)=\frac{t^{-\beta}}{\Gamma(1-\beta)}$, where $0<\beta<1$. More examples of kernels $M$ and $k$ occurring in different physical models are given in Subsection 6.1 of this paper. 
Let $T, l>0$. Firstly, we formulate the following initial-boundary value problem (direct problem) for the state function $u$ :

$$
\begin{aligned}
& D_{t}^{\{k\}}\left(u-u_{0}\right)(t, x)=\left[a(x) u_{x}(t, x)\right]_{x}+f(t, x), x \in(0, l), t \in(0, T), \\
& a(0) u_{x}(t, 0)=h_{0}(t), a(l) u_{x}(t, l)=h_{l}(t), t \in(0, T), \\
& u(0, x)=u_{0}, x \in(0, l),
\end{aligned}
$$

where $a, f, h_{0}, h_{l}$ are given functions and $u_{0}$ is a given number.

The boundary conditions (5) are related to fluxes at $x=0$ and $x=l$. In view of (1), the outward pointing fluxes at $x=0$ and $x=l$ are $H_{0}=a(0) \frac{\partial}{\partial t} M * u(\cdot, 0)$ and $H_{l}=-a(l) \frac{\partial}{\partial t} M * u(\cdot, l)$, respectively. The functions $h_{0}$ and $h_{l}$ involved in (5) can be expressed via fluxes as $h_{0}=k * H_{0}$ and $h_{l}=-k * H_{l}$, respectively.

Next, let us suppose that the diffusivity coefficient $a$ is unknown but the state is specified at the final time $t=T$, i.e.

$$
u(T, x)=u_{T}(x), x \in(0, l),
$$

where $u_{T}$ is a given function. We pose the inverse problem to determine a pair of functions $(a, u)$ that satisfy the conditions (4)-(7).

The inverse problem (4)-(7) will be the main research topic of this paper. We will be able to study it in the case of the constant initial state. Therefore, the quantity $u_{0}$ was defined as a number already in the formulation of the corresponding direct problem.

\section{PRELIMINARIES}

\subsection{Abstract functional spaces and Sonine kernels}

Let $X$ be a Banach space. We define some spaces of abstract functions that map the intervals $(0, T)$ and $[0, T]$ into $X$.

By $L_{p}((0, T) ; X), p \in[1, \infty]$, we denote the abstract Lebesgue spaces, i.e.

$$
\begin{aligned}
& L_{p}((0, T) ; X)=\left\{v:(0, T) \rightarrow X:\|v\|_{L_{p}((0, T) ; X)}:=\left[\int_{0}^{T}\|v(t)\|_{X}^{p} d t\right]^{\frac{1}{p}}<\infty\right\}, p \in[1, \infty), \\
& L_{\infty}((0, T) ; X)=\left\{v:(0, T) \rightarrow X:\|v\|_{L_{\infty}((0, T) ; X)}:=\text { ess } \sup _{t \in(0, T)}\|v(t)\|_{X}<\infty\right\} .
\end{aligned}
$$

The space $C([0, T] ; X)$ contains functions $v:[0, T] \rightarrow X$ that are continuous on $[0, T]$. This is a Banach space with the norm $\|v\|_{C([0, T] ; X)}=\max _{t \in[0, T]}\|v(t)\|_{X}$. Moreover, we define

$$
C_{0}([0, T] ; X)=\{v \in C([0, T] ; X): v(0)=0\}
$$

and

$$
C^{1}([0, T] ; X)=\left\{v: v, v^{\prime} \in C([0, T] ; X)\right\} .
$$

Let $0<\alpha<1$. The abstract Hölder spaces with their norms are defined by

$$
\begin{aligned}
& C_{0}^{\alpha}([0, T] ; X)=\left\{v \in C_{0}([0, T] ; X):\|v\|_{C_{0}^{\alpha}([0, T] ; X)}:=\sup _{0<t_{1}<t_{2}<T} \frac{\left\|v\left(t_{2}\right)-v\left(t_{1}\right)\right\|_{X}}{\left(t_{2}-t_{1}\right)^{\alpha}}<\infty\right\}, \\
& C^{\alpha}([0, T] ; X)=C_{0}^{\alpha}([0, T] ; X)+X=\left\{v: v(t)=v_{1}(t)+v_{2}, v_{1} \in C_{0}^{\alpha}([0, T] ; X), v_{2} \in X\right\}, \\
& \|v\|_{C^{\alpha}([0, T] ; X)}=\|v-v(0)\|_{C_{0}^{\alpha}([0, T] ; X)}+\|v(0)\|_{X} .
\end{aligned}
$$


We also introduce the following space:

$$
C_{0}^{1+\alpha}([0, T] ; X)=\left\{v: v, v^{\prime} \in C_{0}^{\alpha}([0, T] ; X)\right\}, 0<\alpha<1 .
$$

A function $M \in L_{1, l o c}(0, \infty)$ is called the Sonine kernel if the equation

$$
M * k(t)=1, \quad t>0,
$$

has a solution $k \in L_{1, l o c}(0, \infty)$ [19]. The solution $k$, if it exists, is unique [5] and is referred to as associate to $M$. Since the convolution is commutative, $k$ is also the Sonine kernel and $M$ is its associate.

The Sonine kernel is unbounded at $t=0$, because otherwise $M * k(t) \rightarrow 0$ as $t \rightarrow 0^{+}$and this contradicts $M * k(t) \equiv 1$.

Let $M$ be the Sonine kernel and $k$ its associate. Then

$$
D_{t}^{\{k\}}(M * v)=\frac{d}{d t} k * M * v=\frac{d}{d t} 1 * v=v, \quad \forall v \in L_{1}((0, T) ; X) .
$$

Therefore, the operator $M *$ is a one-to-one mapping from $L_{1}((0, T) ; X)$ to the space

$$
M * L_{1}((0, T) ; X)=\left\{M * v: v \in L_{1}((0, T) ; X)\right\}
$$

and $D_{t}^{\{k\}}$ is the inverse of $M *$. The reversed relation to (9) is

$$
M *\left(D_{t}^{\{k\}} v\right)=v, \quad \forall v \in M * L_{1}((0, T) ; X) .
$$

Next, we define some abstract $C$ - and Hölder spaces related to the Sonine kernel $M$ and its associate $k$ :

$$
\begin{aligned}
& C_{0}^{\{k\}}([0, T] ; X):=M * C([0, T] ; X),\|v\|_{C_{0}^{\{k\}}([0, T] ; X)}=\left\|D_{t}^{\{k\}} v\right\|_{C([0, T] ; X)}, \\
& C^{\{k\}}([0, T] ; X):=C_{0}^{\{k\}}([0, T] ; X)+X,\|v\|_{C^{\{k\}}([0, T] ; X)}=\|v-v(0)\|_{C_{0}^{\{k\}}([0, T] ; X)}+\|v(0)\|_{X}, \\
& C_{0}^{\{k\}, \alpha}([0, T] ; X)=M * C_{0}^{\alpha}([0, T] ; X),\|v\|_{C_{0}^{\{k\}, \alpha}([0, T] ; X)}=\left\|D_{t}^{\{k\}} v\right\|_{C_{0}^{\alpha}([0, T] ; X)} .
\end{aligned}
$$

The following continuous embeddings are valid [9]:

$$
\begin{aligned}
& C^{1}([0, T] ; X) \hookrightarrow C^{\{k\}}([0, T] ; X) \hookrightarrow C([0, T] ; X) \\
& C_{0}^{1+\alpha}([0, T] ; X) \hookrightarrow C_{0}^{\{k\}, \alpha}([0, T] ; X) \hookrightarrow C_{0}^{\alpha}([0, T] ; X) .
\end{aligned}
$$

Clearly, the integration improves the regularity of a function. Therefore, one may ask the question: does the subspace $C_{0}^{\{k\}, \alpha}([0, T] ; X)$ of $C_{0}^{\alpha}([0, T] ; X)$ consist of functions that are Hölder continuous of an order greater than $\alpha$ ? This is true provided the function $M$ satisfies certain additional restrictions, as can be seen from the following lemma.

Lemma 1. [9] If $M(t) \leq c_{1} t^{\beta-1},\left|M^{\prime}(t)\right| \leq c_{2} t^{\beta-2}, t \in(0, T)$ for some $c_{1}, c_{2}>0,0<\beta \leq \alpha<1$, then $M * \in \mathscr{B}\left(C_{0}^{\alpha-\beta}([0, T] ; X), C_{0}^{\alpha}([0, T] ; X)\right)$, which implies $C_{0}^{\{k\}, \alpha-\beta}([0, T] ; X) \hookrightarrow C_{0}^{\alpha}([0, T] ; X)$.

In the particular case $M(t)=\frac{t^{\beta-1}}{\Gamma(\beta)}$ we have $C_{0}^{\{k\}, \alpha-\beta}([0, T] ; X)=C_{0}^{\alpha}([0, T] ; X)[6]$. 


\subsection{Inverse source problem}

In this section we formulate the results regarding an inverse source problem that consists in finding the pair $(\phi, w)$ satisfying

$$
\left.\begin{array}{l}
D_{t}^{\{k\}} w(t, x)=a(x) w_{x x}(t, x)+\phi(x) r(t, x)+q(t, x), x \in(0, l), t \in(0, T), \\
w(t, 0)=w(t, l)=0, t \in(0, T), \\
w(0, x)=0, x \in(0, l), \\
w(T, x)=w_{T}(x), x \in(0, l),
\end{array}\right\}
$$

where $a, r, q, w_{T}$ are given functions. Such a problem is an important tool in the analysis of the inverse diffusivity problem (4)-(7) posed in Section 2.

Theorem 1. [9] Let $k$ be the Sonine kernel and $M$ its associate. Assume that

$$
\begin{aligned}
& k \in C(0, \infty), k \text { is nonincreasing, } \exists t_{k}>0: k(t) \text { is strictly decreasing in }\left(0, t_{k}\right), \\
& M \in C^{1}(0, \infty), M>0, M^{\prime} \leq 0,-M^{\prime} \text { is nonincreasing and convex. }
\end{aligned}
$$

Moreover, let $a \in C[0, T], a(x)>0, x \in[0, l]$, and one of the following assumptions be valid:

(A1) $r \in C_{0}^{1+\alpha_{1}}([0, T] ; C[0, l])$ for some $0<\alpha_{1}<1$;

(A2) $r \in C_{0}^{\{k\}, \alpha_{1}}([0, T] ; C[0, l])$ and $M(t) \geq c t^{\gamma-1}, t \in(0, T)$, for some $c>0,0<\gamma<\alpha_{1}<1$;

(A3) $r \in C_{0}^{\{k\}, \alpha_{1}-\beta}([0, T] ; C[0, l])$ and $c_{1} t^{\gamma-1} \leq M(t) \leq c_{2} t^{\beta-1},\left|M^{\prime}(t)\right| \leq c_{3} t^{\beta-2}, t \in(0, T)$,

for some $c_{1}, c_{2}, c_{3}>0,0<\beta \leq \gamma<\alpha_{1}<1$.

Additionally, we assume that

$$
\begin{aligned}
& r \geq 0, \quad D_{t}^{\{k\}} r \geq 0 \\
& \text { a.e. } x \in(0, l) \exists t_{x} \in(0, T]: r\left(t_{x}, x\right)>0, \\
& \text { for any } b \in\{0 ; l\} \text { either } r(T, b)>0 \text { or } r(\cdot, b)=0 .
\end{aligned}
$$

Finally, let $(\phi, w) \in C[0, l] \times C_{0}^{\{k\}}([0, T] ; C[0, l]) \cap C_{0}\left([0, T] ; W_{p}^{2}(0, l)\right)$ for some $p>1$ solve $(11)$ for $q=0$, $w_{T}=0$. Then $(\phi, w)=(0,0)$.

Theorem 2. [9] Let $k$ be the Sonine kernel, $M$ its associate and (12), (13) hold. Let $r$ and $M$ satisfy one of the assumptions (A1)-(A3), the inequalities (14) and $r(T, x)>0, x \in[0, l]$. If $w_{T} \in C^{2}[0, l], w_{T}(0)=w_{T}(l)=0$, $q \in C_{0}^{\{k\}, \alpha_{2}}\left([0, T] ; L_{p}(0, l)\right) \cap C_{0}([0, T] ; C[0, l])$ for some $p>1$ and $0<\alpha_{2}<1$, then (11) has a unique solution $(\phi, w)$ in the space $C[0, l] \times C_{0}^{\{k\}, \alpha^{\prime}}\left([0, T] ;{ }_{0} W_{p}^{2}(0, l)\right) \cap C_{0}\left([0, T] ; C^{2}[0, l]\right)$, where $\alpha^{\prime}=\min \left\{\hat{\alpha} ; \alpha_{2}\right\}$,

$$
\hat{\alpha}= \begin{cases}\alpha_{1} & \text { in cases }(\mathrm{A} 1),(\mathrm{A} 2) \\ \alpha_{1}-\beta & \text { in case }(\mathrm{A} 3)\end{cases}
$$

and ${ }_{0} W_{p}^{2}(0, l)=\left\{z \in W_{p}^{2}(0, l): z(0)=z(l)=0\right\}$. Moreover, the estimate

$$
\begin{aligned}
& \|\phi\|_{C[0, l]}+\|w\|_{C_{0}^{\{k\}, \alpha^{\prime}}\left([0, T] ; 0 W_{p}^{2}(0, l)\right) \cap C_{0}\left([0, T] ; C^{2}[0, l]\right)} \\
& \leq C_{1}\left(\|q\|_{C_{0}^{\{k\}, \alpha_{2}\left([0, T] ; L_{p}(0, l)\right) \cap C_{0}([0, T] ; C[0, l])}}+\left\|w_{T}\right\|_{C^{2}[0, l]}\right)
\end{aligned}
$$

is valid where the constant $C_{1}$ is independent of $q$ and $w_{T}$. 


\section{STATEMENTS ABOUT THE DIRECT PROBLEM (4)-(6)}

Lemma 2. Let $k$ be the Sonine kernel and $M$ its associate. Let $a \in C[0, l]$. If a function $u \in C^{\{k\}}\left([0, T] ; L_{1}(0, l)\right)$ $\cap C\left([0, T] ; W_{1}^{1}(0, l)\right)$ satisfies $a u_{x} \in C_{0}\left([0, T] ; W_{1}^{1}(0, l)\right)$ and solves (4)-(6), then

$$
\int_{0}^{l} u(t, y) d y=M *\left[h_{l}-h_{0}+\int_{0}^{l} f(\cdot, y) d y\right](t)+l u_{0}, t \in[0, T] .
$$

Proof. Integrating (4) from 0 to $l$ and taking into account (5), we obtain $D_{t}^{\{k\}}\left[\int_{0}^{l} u(t, y) d y-l u_{0}\right]=h_{l}(t)-$ $h_{0}(t)+\int_{0}^{l} f(t, y) d y$. Applying the operator $M *$ to this relation and taking into account (10), we reach (19).

Remark 1. The relation (19) is the integral conservation law. Indeed, in Section 2 we saw that $f=k * F$, $h_{0}=k * H_{0}$ and $h_{l}=-k * H_{l}$, where $F$ is the physical source function and $H_{0}$ and $H_{l}$ are the outward pointing fluxes. Therefore, since $M * k=1$, the relation (19) can be rewritten as

$$
\int_{0}^{l} u(t, y) d y=-\int_{0}^{t}\left[H_{l}(\tau)+H_{0}(\tau)\right] d \tau+\int_{0}^{t} \int_{0}^{l} F(\tau, y) d y d \tau+\int_{0}^{l} u_{0} d x .
$$

Let us define an operator $\mathscr{J}: L_{1}(0, l) \rightarrow\left\{z \in W_{1}^{1}(0, l): z(0)=z(l)=0\right\}$ by the following formula:

$$
\mathscr{J} \rho(x)=\frac{l-x}{l} \int_{0}^{x} \rho(y) d y+\frac{x}{l} \int_{l}^{x} \rho(y) d y, x \in[0, l] .
$$

Proposition 1. Let $k$ be the Sonine kernel and $M$ its associate. Let $a \in C[0, l]$. Then the following assertions are valid.

(i) If a function $u \in C^{\{k\}}\left([0, T] ; L_{1}(0, l)\right) \cap C\left([0, T] ; W_{1}^{1}(0, l)\right)$ satisfies a $u_{x} \in C_{0}\left([0, T] ; W_{1}^{1}(0, l)\right)$ and solves (4)-(6), then the function $v \in C_{0}^{\{k\}}\left([0, T] ; W_{1}^{1}(0, l)\right) \cap C_{0}\left([0, T] ; W_{1}^{2}(0, l)\right)$ defined by

$$
v(t, x)=\mathscr{J} u(t, x)
$$

satisfies $a v_{x x} \in C_{0}\left([0, T] ; W_{1}^{1}(0, l)\right)$ and solves the following problem:

$$
\begin{aligned}
& D_{t}^{\{k\}} v(t, x)=a(x) v_{x x}(t, x)+g(t, x), x \in(0, l), t \in(0, T), \\
& v(t, 0)=v(t, l)=0, t \in(0, T), \\
& v(0, x)=0, x \in(0, l),
\end{aligned}
$$

where

$$
g(t, x)=\mathscr{J} f(t, x)-\frac{l-x}{l} h_{0}(t)-\frac{x}{l} h_{l}(t) .
$$

(ii) If $f \in C\left([0, T] ; L_{1}(0, l)\right), h_{0}, h_{l} \in C_{0}[0, T]$ and a function $v \in C_{0}^{\{k\}}\left([0, T] ; W_{1}^{1}(0, l)\right) \cap C_{0}\left([0, T] ; W_{1}^{2}(0, l)\right)$ satisfies av $x_{x x} \in C_{0}\left([0, T] ; W_{1}^{1}(0, l)\right)$ and solves (22)-(24) with $g$ of the form (25), then the function $u \in C^{\{k\}}\left([0, T] ; L_{1}(0, l)\right) \cap C\left([0, T] ; W_{1}^{1}(0, l)\right)$ defined by

$$
u(t, x)=v_{x}(t, x)+\frac{1}{l} M *\left[h_{l}-h_{0}+\int_{0}^{l} f(\cdot, y) d y\right](t)+u_{0}
$$

satisfies $a_{x} \in C_{0}\left([0, T] ; W_{1}^{1}(0, l)\right)$ and solves (4)-(6). 
Proof. (i) The assertions regarding the regularity of $v$ and the conditions (23), (24) immediately follow from (21), (20), the assumed regularity of $u$ and (6). Applying the operator $\mathscr{J}$ to (4), observing that $\mathscr{J}\left[\left(a u_{x}\right)_{x}\right]=a u_{x}-\frac{l-x}{l} a(0) u_{x}(t, 0)-\frac{x}{l} a(l) u_{x}(t, l)$ and taking the boundary conditions $(5)$ and the relation $u_{x}=v_{x x}$ into account, we obtain (22) with $g$ of the form (25).

(ii) The asserted regularity of $u$ and the condition (6) follow from the formula (26), the assumed regularity of $f, h$ and $v$ and the relation (24). Passing to the limit $x \rightarrow 0^{+}$in (22) and observing (23), (25), we have $0=a(0) v_{x x}(t, 0)-h_{0}(t)$. Since $v_{x x}=u_{x}$, we obtain the boundary condition $a(0) u_{x}(t, 0)=h_{0}(t)$. In a similar manner we prove another boundary condition $a(l) u_{x}(t, l)=h_{l}(t)$, too. Next, we apply the operator $\frac{\partial}{\partial x}$ to the equation (22). Replacing $v_{x}$ by $u-u_{0}-\frac{1}{l} M *\left[h_{l}-h_{0}+\int_{0}^{l} f(\cdot, y) d y\right]$ in the left-hand side of the obtained equation and using (9), we have

$$
D_{t}^{\{k\}}\left(u-u_{0}\right)(t, x)-Q(t)=\left(a(x) v_{x x}(t, x)\right)_{x}+g_{x}(t, x),
$$

where $Q(t)=\frac{1}{l}\left[h_{l}(t)-h_{0}(t)+\int_{0}^{l} f(t, y) d y\right]$. On the other hand, (25) implies $g_{x}(t, x)=f(t, x)-Q(t)$. Therefore, since $v_{x x}=u_{x}$, we obtain the equation (4).

\section{RESULTS ABOUT THE INVERSE PROBLEM (4)-(7)}

Firstly, we prove the global uniqueness of the solution to (4)-(7).

Theorem 3. Let $k$ be the Sonine kernel, $M$ its associate and (12), (13) hold. Let the inverse problem (4)-(7) have two solutions $(a, u),\left(a_{1}, u_{1}\right) \in C[0, l] \times C^{\{k\}}\left([0, T] ; L_{1}(0, l)\right) \cap C\left([0, T] ; W_{1}^{1}(0, l)\right)$ such that a $u_{x}, a_{1} u_{1, x} \in$ $C_{0}\left([0, T] ; W_{1}^{1}(0, l)\right)$ and $u_{1}-u \in C\left([0, T] ; W_{p}^{1}(0, l)\right)$ for some $p>1$. Assume that $a_{1}(x)>0, x \in[0, l]$, and one of the following conditions is valid:

(A4) $u_{x} \in C_{0}^{1+\alpha_{1}}([0, T] ; C[0, l])$ for some $0<\alpha_{1}<1$;

(A5) $u_{x} \in C_{0}^{\{k\}, \alpha_{1}}([0, T] ; C[0, l])$ and $M(t) \geq c t^{\gamma-1}, t \in(0, T)$, for some $c>0,0<\gamma<\alpha_{1}<1$;

(A6) $u_{x} \in C_{0}^{\{k\}, \alpha_{1}-\beta}([0, T] ; C[0, l])$ and $c_{1} t^{\gamma-1} \leq M(t) \leq c_{2} t^{\beta-1},\left|M^{\prime}(t)\right| \leq c_{3} t^{\beta-2}, t \in(0, T)$, for some $c_{1}, c_{2}, c_{3}>0,0<\beta \leq \gamma<\alpha_{1}<1$.

Additionally, let

$$
\begin{aligned}
& u_{x} \geq 0, \quad D_{t}^{\{k\}} u_{x} \geq 0, \\
& \text { a.e. } x \in(0, l) \exists t_{x} \in(0, T]: u_{x}\left(t_{x}, x\right)>0, \\
& \text { for any } b \in\{0 ; l\} \text { either } u_{x}(T, b)>0 \text { or } u_{x}(\cdot, b)=0 .
\end{aligned}
$$

Then $(a, u)=\left(a_{1}, u_{1}\right)$.

Proof. Let us denote $v(t, x)=\mathscr{J} u(t, x)$ and $v_{1}(t, x)=\mathscr{J} u_{1}(t, x)$. Due to Proposition 1 (i), $v$ is a solution to the problem (22)-(24) and $v_{1}$ is a solution to the following problem:

$$
\begin{aligned}
& D_{t}^{\{k\}} v_{1}(t, x)=a_{1}(x) v_{1, x x}(t, x)+g(t, x), x \in(0, l), t \in(0, T), \\
& v_{1}(t, 0)=v_{1}(t, l)=0, t \in(0, T), \\
& v_{1}(0, x)=0, x \in(0, l) .
\end{aligned}
$$

Moreover, $v(T, x)=v_{1}(T, x)$ and $v_{x x}=u_{x}, v_{1, x x}=u_{1, x}$. The pair of differences $(\phi, w)=\left(a_{1}-a, v_{1}-v\right)$ belongs to $C[0, l] \times C_{0}^{\{k\}}\left([0, T] ; W_{1}^{1}(0, l)\right) \cap C_{0}\left([0, T] ; W_{p}^{2}(0, l)\right)$ and is a solution to the following problem:

$$
\begin{aligned}
& D_{t}^{\{k\}} w(t, x)=a_{1}(x) w_{x x}(t, x)+\phi(x) u_{x}(t, x), x \in(0, l), t \in(0, T), \\
& w(t, 0)=w(t, l)=0, t \in(0, T), \\
& w(0, x)=0, x \in(0, l) \\
& w(T, x)=0, x \in(0, l) .
\end{aligned}
$$


This is the inverse source problem (11) with the data $r=u_{x}, q=0$ and $w_{T}=0$. The assumptions of Theorem 1 are satisfied for this problem. Theorem 1 implies $\phi=0$ and $w=0$. Thus, $a=a_{1}$ and $v=v_{1}$.

Due to the assumed regularity of $a$ and $u$ the functions $f, h_{0}$ and $h_{l}$ satisfy $f \in C\left([0, T] ; L_{1}(0, l)\right)$ and $h_{0}, h_{l} \in C_{0}[0, T]$. Therefore, we can apply Proposition 1 (ii) for the problems (22)-(24) and (30)-(32). We have $u=v_{x}+\frac{1}{l} M *\left[h_{l}-h_{0}+\int_{0}^{l} f(\cdot, y) d y\right]+u_{0}$ and $u_{1}=v_{1, x}+\frac{1}{l} M *\left[h_{l}-h_{0}+\int_{0}^{l} f(\cdot, y) d y\right]+u_{0}$. Since $v=v_{1}$, we obtain $u=u_{1}$. This completes the proof.

Next, we are going to establish the local existence and stability for the inverse coefficient problem. Let us formulate a problem that contains approximate data:

$$
\begin{aligned}
& D_{t}^{\{k\}}\left(\widetilde{u}-\widetilde{u}_{0}\right)(t, x)=\left[\widetilde{a}(x) \widetilde{u}_{x}(t, x)\right]_{x}+\widetilde{f}(t, x), x \in(0, l), t \in(0, T), \\
& \widetilde{a}(0) \widetilde{u}_{x}(t, 0)=\widetilde{h}_{0}(t), \widetilde{a}(l) \widetilde{u}_{x}(t, l)=\widetilde{h}_{l}(t), t \in(0, T), \\
& \widetilde{u}(0, x)=\widetilde{u}_{0}, x \in(0, l), \\
& \widetilde{u}(T, x)=\widetilde{u}_{T}(x), x \in(0, l) .
\end{aligned}
$$

Let us denote the data vectors of the exact and approximate problems as follows:

$$
D=\left(f, h_{0}, h_{l}, u_{0}, u_{T}\right), \quad \widetilde{D}=\left(\widetilde{f}, \widetilde{h}_{0}, \widetilde{h}_{l}, \widetilde{u}_{0}, \widetilde{u}_{T}\right) .
$$

The aim is to show that (34)-(37) has a solution that is close to a solution to (4)-(7) provided the difference of $\widetilde{D}$ and $D$ is sufficiently small.

Theorem 4. Let $k$ be the Sonine kernel, $M$ its associate and (12), (13) hold. Let (4)-(7) have a solution $(a, u) \in C[0, l] \times C^{\{k\}}\left([0, T] ; L_{1}(0, l)\right) \cap C\left([0, T] ; W_{1}^{1}(0, l)\right)$ such that $a u_{x} \in C_{0}\left([0, T] ; W_{1}^{1}(0, l)\right), a(x)>0, x \in$ $[0, l], u_{x}$ and $M$ satisfy one of the assumptions (A4)-(A6), the inequalities (27) and $u_{x}(T, x)>0, x \in[0, l]$. Assume that $\widetilde{f} \in C\left([0, T] ; L_{1}(0, l)\right), \widetilde{h}_{0}, \widetilde{h}_{l} \in C_{0}[0, T], \widetilde{u}_{T} \in C[0, l]$ and

$$
\int_{0}^{l} \widetilde{u}_{T}(y) d y=M *\left[\widetilde{h}_{l}-\widetilde{h}_{0}+\int_{0}^{l} \widetilde{f}(\cdot, y) d y\right](T)+l \widetilde{u}_{0} .
$$

Let $0<\alpha_{2}<1$ and $p>1$. Then there exists a constant $\delta>0$, depending on $k, a, u, \alpha_{2}, p$ such that if $\widetilde{D}-D \in \mathscr{D} \quad$ and $\quad\|\widetilde{D}-D\|_{\mathscr{D}} \leq \delta \quad$ where $\mathscr{D}=C_{0}^{\{k\}, \alpha_{2}}\left([0, T] ; L_{1}(0, l)\right) \times\left(C_{0}^{\{k\}, \alpha_{2}}[0, T]\right)^{2} \times \mathbb{R} \times C^{1}[0, l]$, then there exist functions $\widetilde{a} \in C[0, l]$ and $\widetilde{u} \in C^{\{k\}}\left([0, T] ; L_{1}(0, l)\right) \cap C\left([0, T] ; W_{1}^{1}(0, l)\right)$ such that $\widetilde{a} \widetilde{u}_{x} \in C_{0}\left([0, T] ; W_{1}^{1}(0, l)\right)$ and the pair $(\widetilde{a}, \widetilde{u})$ is a solution to the problem (34)-(37). Moreover, $\widetilde{u}-u \in$ $\left(C_{0}^{\{k\}, \alpha^{\prime}}\left([0, T] ; W_{p}^{1}(0, l)\right)+\mathbb{R}\right) \cap C\left([0, T] ; C^{1}[0, l]\right)$ and the following estimate is valid:

$$
\begin{aligned}
& \|\widetilde{a}-a\|_{C[0, l]}+\|\widetilde{u}-u\|_{\left(C_{0}^{\{k\}, \alpha^{\prime}}\left([0, T] ; W_{p}^{1}(0, l)\right)+\mathbb{R}\right) \cap C\left([0, T] ; C^{1}[0, l]\right)} \\
& +\left\|\widetilde{a} \widetilde{u}_{x}-a u_{x}\right\|_{C_{0}\left([0, T] ; W_{1}^{1}(0, l)\right)} \leq K\|\widetilde{D}-D\|_{\mathscr{D}}
\end{aligned}
$$

where $\alpha^{\prime}=\min \left\{\hat{\alpha} ; \alpha_{2}\right\}, \hat{\alpha}=\left\{\begin{array}{ll}\alpha_{1} & \text { in cases (A4), (A5) } \\ \alpha_{1}-\beta & \text { in case (A6) }\end{array}\right.$ and the constant $K$ is independent of $\widetilde{D}-D$.

Proof. Let us consider the problem to find a pair $(\phi, w)$ that satisfies the following relations:

$$
\begin{aligned}
& D_{t}^{\{k\}} w(t, x)=a(x) w_{x x}(t, x)+\phi(x) u_{x}(t, x)+\phi(x) w_{x x}(t, x)+q(t, x), x \in(0, l), t \in(0, T), \\
& w(t, 0)=w(t, l)=0, t \in(0, T), \\
& w(0, x)=0, x \in(0, l) \\
& w(T, x)=w_{T}(x), x \in(0, l)
\end{aligned}
$$


where $q(t, x)=\mathscr{J}(\widetilde{f}-f)(t, x)-\frac{l-x}{l}\left(\widetilde{h}_{0}-h_{0}\right)(t)-\frac{x}{l}\left(\widetilde{h}_{l}-h_{l}\right)(t)$ and $w_{T}(x)=\mathscr{J}\left(\widetilde{u}_{T}-u_{T}\right)(x)$. In case $\widetilde{D}-D \in \mathscr{D}$, we have $q \in C_{0}^{\{k\}, \alpha_{2}}\left([0, T] ; W_{1}^{1}(0, T)\right), w_{T} \in C^{2}[0, T], w_{T}(0)=w_{T}(l)=0$ and

$$
\|q\|_{C_{0}^{\{k\}, \alpha_{2}\left([0, T] ; W_{1}^{1}(0, T)\right)}}+\left\|w_{T}\right\|_{C^{2}[0, l]} \leq C_{2}\|\widetilde{D}-D\|_{\mathscr{D}}
$$

with some constant $C_{2}>0$. Further, let $\mathscr{F}_{a, r}$ stand for the $a$ - and $r$-dependent operator that maps the pair of functions $\left(q, w_{T}\right)$ to the solution of the inverse source problem (11). Let us fix some $p>1$. Due to Theorem 2 , the problem (40)-(43) is in the space

$$
\mathscr{W}=C[0, l] \times C_{0}^{\{k\}, \alpha^{\prime}}\left([0, T] ;{ }_{0} W_{p}^{2}(0, l)\right) \cap C_{0}\left([0, T] ; C^{2}[0, l]\right)
$$

equivalent to the following operator equation:

$$
S=F(S), \quad \text { where } \quad S=(\phi, w), F(S)=\mathscr{F}_{a, u_{x}}\left(\phi w_{x x}+q, w_{T}\right) .
$$

Using (18) and (44), we deduce the estimate

$$
\|F(S)\|_{\mathscr{W}} \leq C_{1}\left(\left\|\phi w_{x x}+q\right\|_{C_{0}^{\{k\}, \alpha_{2}}\left([0, T] ; L_{p}(0, l)\right) \cap C_{0}([0, T] ; C[0, l])}+\left\|w_{T}\right\|_{C^{2}[0, l]}\right) \leq C_{3}\|S\|_{\mathscr{W}}^{2}+C_{4}\|\widetilde{D}-D\|_{\mathscr{D}},
$$

where $C_{3}=C_{1} \max \left\{\omega_{1} ; 1\right\}, C_{4}=C_{1} C_{2} \max \left\{\omega_{2} ; 1\right\}$ and $\omega_{1}$ and $\omega_{2}$ are the norms of the embedding operators $C_{0}^{\{k\}, \alpha^{\prime}}\left([0, T] ; L_{p}(0, l)\right) \hookrightarrow C_{0}^{\{k\}, \alpha_{2}}\left([0, T] ; L_{p}(0, l)\right)$ and $C_{0}^{\{k\}, \alpha_{2}}\left([0, T] ; W_{1}^{1}(0, l)\right) \hookrightarrow C_{0}^{\{k\}, \alpha_{2}}\left([0, T] ; L_{p}(0, l)\right)$ $\cap C_{0}([0, T] ; C[0, l])$, respectively. Similarly, for $S_{j}=\left(\phi_{j}, w_{j}\right), j=1,2$, we have

$$
\begin{aligned}
& \left\|F\left(S_{1}\right)-F\left(S_{2}\right)\right\|_{\mathscr{W}}=\left\|\mathscr{F}_{a, u_{x}}\left(\left(\phi_{1}-\phi_{2}\right) w_{1, x x}+\phi_{2}\left(w_{1, x x}-w_{2, x x}\right), 0\right)\right\|_{\mathscr{W}} \\
& \leq C_{3}\left(\left\|S_{1}\right\|_{\mathscr{W}}+\left\|S_{2}\right\|_{\mathscr{W}}\right)\left\|S_{1}-S_{2}\right\|_{\mathscr{W}}
\end{aligned}
$$

Let $\|\widetilde{D}-D\|_{\mathscr{D}} \leq \delta=\frac{1}{8 C_{3} C_{4}}$. Then, for any $S$ such that $\|S\|_{\mathscr{W}} \leq \rho=K_{0}\|\widetilde{D}-D\|_{\mathscr{D}}$ where $K_{0}=2 C_{4}$ from (46), we have

$$
\begin{aligned}
& \|F(S)\|_{\mathscr{W}} \leq C_{3} \rho^{2}+C_{4}\|\widetilde{D}-D\|_{\mathscr{D}}=C_{3} K_{0}^{2}\|\widetilde{D}-D\|_{\mathscr{D}}^{2}+C_{4}\|\widetilde{D}-D\|_{\mathscr{D}}=C_{3} K_{0}^{2}\|\widetilde{D}-D\|_{\mathscr{D}}\|\widetilde{D}-D\|_{\mathscr{D}} \\
& +C_{4}\|\widetilde{D}-D\|_{\mathscr{D}} \leq C_{3} K_{0}^{2} \delta\|\widetilde{D}-D\|_{\mathscr{D}}+C_{4}\|\widetilde{D}-D\|_{\mathscr{D}}=\frac{3 K_{0}}{4}\|\widetilde{D}-D\|_{\mathscr{D}}<\rho .
\end{aligned}
$$

This implies that the operator $F$ leaves the ball $\|S\|_{\mathscr{W}} \leq \rho$ invariant. Further, for any $S_{j}, j=1,2$ such that $\left\|S_{j}\right\|_{\mathscr{W}} \leq \rho$ from (47), we obtain

$$
\begin{aligned}
& \left\|F\left(S_{1}\right)-F\left(S_{2}\right)\right\|_{\mathscr{W}} \leq 2 C_{3} \rho\left\|S_{1}-S_{2}\right\|_{\mathscr{W}}=2 C_{3} K_{0}\|\widetilde{D}-D\|_{\mathscr{D}}\left\|S_{1}-S_{2}\right\|_{\mathscr{W}} \\
& \leq 2 C_{3} K_{0} \delta\left\|S_{1}-S_{2}\right\|_{\mathscr{W}}^{2}=\frac{1}{2}\left\|S_{1}-S_{2}\right\|_{\mathscr{W}}
\end{aligned}
$$

This shows that the operator $F$ is a contraction in the ball $\|S\|_{\mathscr{W}} \leq \rho$. Consequently, the equation (45) and the equivalent problem (40)-(43) have a unique solution $S=(\phi, w)$ in such a ball. Due to the definition of $\rho$ we have the estimate

$$
\|\phi\|_{C[0, l]}+\|w\|_{C_{0}^{\{k\}, \alpha^{\prime}}\left([0, T] ; 0 W_{p}^{2}(0, l)\right) \cap C_{0}\left([0, T] ; C^{2}[0, l]\right)} \leq K_{0}\|\widetilde{D}-D\|_{\mathscr{D}}
$$

Let $v=\mathscr{J} u$. By Proposition 1 (i), $v$ belongs to $C_{0}^{\{k\}}\left([0, T] ; W_{1}^{1}(0, l)\right) \cap C_{0}\left([0, T] ; W_{1}^{2}(0, l)\right)$, satisfies $a v_{x x} \in C_{0}\left([0, T] ; W_{1}^{1}(0, l)\right)$ and is a solution to (22)-(24), where $g$ is given by (25). Further, let $\widetilde{v}=v+w$. Due to the properties of $v$ and $w$ we have $\widetilde{v} \in C_{0}^{\{k\}}\left([0, T] ; W_{1}^{1}(0, l)\right) \cap C_{0}\left([0, T] ; W_{1}^{2}(0, l)\right)$. Adding the equations 
(40), (41) and (42) to the equations (22), (23) and (24), respectively, and replacing $u_{x}$ by $v_{x x}$, we see that $\widetilde{v}$ is a solution to the following problem:

$$
\begin{aligned}
& D_{t}^{\{k\}} \widetilde{v}(t, x)=\widetilde{a}(x) \widetilde{v}_{x x}(t, x)+\widetilde{g}(t, x), x \in(0, l), t \in(0, T), \\
& \widetilde{v}(t, 0)=\widetilde{v}(t, l)=0, t \in(0, T) \\
& \widetilde{v}(0, x)=0, x \in(0, l)
\end{aligned}
$$

where $\widetilde{a}=a+\phi$ and

$$
\widetilde{g}(t, x)=g(t, x)+q(t, x)=\mathscr{J} \widetilde{f}(t, x)-\frac{l-x}{l} \widetilde{h}_{0}(t)-\frac{x}{l} \widetilde{h}_{l}(t)
$$

Since $\widetilde{v} \in C_{0}^{\{k\}}\left([0, T] ; W_{1}^{1}(0, l)\right)$ and $\widetilde{g} \in C_{0}\left([0, T] ; W_{1}^{1}(0, l)\right)$, from the equation (49) we obtain that $\widetilde{a v}_{x x} \in$ $C_{0}\left([0, T] ; W_{1}^{1}(0, l)\right)$. Applying Proposition 1 (ii) to (49)-(51), we obtain that the function $\widetilde{u} \in$ $C^{\{k\}}\left([0, T] ; L_{1}(0, l)\right) \cap C\left([0, T] ; W_{1}^{1}(0, l)\right)$ defined by

$$
\widetilde{u}(t, x)=\widetilde{v}_{x}(t, x)+\frac{1}{l} M *\left[\widetilde{h}_{l}-\widetilde{h}_{0}+\int_{0}^{l} \widetilde{f}(\cdot, y) d y\right](t)+\widetilde{u}_{0}
$$

satisfies $\widetilde{a} \widetilde{u}_{x} \in C_{0}\left([0, T] ; W_{1}^{1}(0, l)\right)$ and solves (34)-(36). Since $v(T, x)=\mathscr{J} u_{T}(x)$ and $w(T, x)=w_{T}(x)=$ $\mathscr{J}\left(\widetilde{u}_{T}-u_{T}\right)(x)$, we have $\widetilde{v}(T, x)=\mathscr{J} \widetilde{u}_{T}(x)$. Hence, from (52) we obtain

$$
\widetilde{u}(T, x)=\widetilde{u}_{T}(x)-\frac{1}{l} \int_{0}^{l} \widetilde{u}_{T}(y) d y+\frac{1}{l} M *\left[\widetilde{h}_{l}-\widetilde{h}_{0}+\int_{0}^{l} \widetilde{f}(\cdot, y) d y\right](T)+\widetilde{u}_{0}
$$

Using (38), we obtain (37). Therefore, $(\widetilde{a}, \widetilde{u})$ is a solution to (34)-(37).

It remains to prove $\widetilde{u}-u \in\left(C_{0}^{\{k\}, \alpha^{\prime}}\left([0, T] ; W_{p}^{1}(0, l)\right)+\mathbb{R}\right) \cap C\left([0, T] ; C^{1}[0, l]\right)$ and the estimate (39). From

$$
\widetilde{u}(t, x)-u(t, x)=w_{x}(t, x)+\frac{1}{l} M *\left[\widetilde{h}_{l}-h_{l}-\widetilde{h}_{0}+h_{0}+\int_{0}^{l}(\widetilde{f}-f)(\cdot, y) d y\right](t)+\widetilde{u}_{0}-u_{0}
$$

in view of $w \in C_{0}^{\{k\}, \alpha^{\prime}}\left([0, T] ;{ }_{0} W_{p}^{2}(0, l)\right) \cap C_{0}\left([0, T] ; C^{2}[0, l]\right)$ and the assumptions on $\widetilde{D}-D$, we have $\widetilde{u}-u \in$ $\left(C_{0}^{\{k\}, \alpha^{\prime}}\left([0, T] ; W_{p}^{1}(0, l)\right)+\mathrm{R}\right) \cap C\left([0, T] ; C^{1}[0, l]\right)$. Next, we note that $\widetilde{a v} \widetilde{v}_{x x}-a v_{x x}=D_{t}^{\{k\}} w+g-\widetilde{g}$. Thus, due to (48) and the definitions of $g$ and $\widetilde{g}$ as well as the norm $\|\cdot\|_{\mathscr{D}}$, we obtain

$$
\left\|\widetilde{a} \widetilde{v}_{x x}-a v_{x x}\right\|_{C_{0}\left([0, T] ; W_{1}^{1}(0, l)\right)} \leq K_{1}\|\widetilde{D}-D\|_{\mathscr{D}}
$$

where $K_{1}$ is a constant. By means of (48), (53), (54) as well as the relations $\phi=\widetilde{a}-a$ and $\widetilde{a v}_{x x}-a v_{x x}=$ $\widetilde{a} \widetilde{u}_{x}-a u_{x}$, we obtain the estimate (39). This completes the proof.

Remark 2. The estimate (39) shows that the solution to (34)-(37) continuously depends on the first order derivative of the measured function $u_{T}$. The degree of ill-posedness of the inverse diffusivity problem (4)-(7) is lower than that of the inverse problem to reconstruct an $x$-dependent source factor from final measurements [9]. The solution to the latter problem continuously depends on the second order derivatives of the final data. 


\section{ADDITIONAL REMARKS}

\subsection{Examples of kernels}

Let us define the following subset of the set of completely monotonic functions:

$$
\mathscr{C} \mathscr{M}=\left\{z \in L_{1, l o c}(0, \infty) \cap C^{\infty}(0, \infty): \lim _{t \rightarrow 0^{+}} z(t)=\infty,(-1)^{i} z^{(i)}(t)>0, t>0, i=0,1,2, \ldots\right\} .
$$

It holds the following statement:

Lemma 3. [4] Let $k \in \mathscr{C} \mathscr{M}$. Then $k$ is the Sonine kernel and its associate $M$ also belongs to $\mathscr{C} \mathscr{M}$.

Clearly, for $k, M \in \mathscr{C} \mathscr{M}$, the conditions (12) and (13) are satisfied.

There are many examples of generalized fractional derivatives with kernels of the class $\mathscr{C} \mathscr{M}$ in models of subdiffusion. Let us list some of them. A more detailed description can be found, e.g., in [10].

- Distributed fractional derivatives. Then

$$
\text { either } k(t)=\int_{0}^{1} \frac{t^{-\beta}}{\Gamma(1-\beta)} d p(\beta) \text { or } M(t)=\int_{0}^{1} \frac{t^{\beta-1}}{\Gamma(\beta)} d p(\beta)
$$

where $p$ is a Borel measure. Such derivatives occur in the modelling of accelerating and retarding subdiffusion, also of ultraslow diffusion [12]. A particular case is the multiterm derivatives when

$$
\text { either } k(t)=\sum_{j=1}^{s} \varkappa_{j} \frac{t^{-\beta_{j}}}{\Gamma\left(1-\beta_{j}\right)} \quad \text { or } \quad M(t)=\sum_{j=1}^{s} \varkappa_{j} \frac{t^{\beta_{j}-1}}{\Gamma\left(\beta_{J}\right)}, \quad \text { where } \quad 0<\beta_{1}<\ldots<\beta_{s}<1, \quad \varkappa_{j}>0 .
$$

- Tempered fractional derivatives. They are used in the modelling of slow transition from anomalous diffusion to the normal one. We can point out three different cases that occur in the literature:

$$
M(t)=\frac{1}{\Gamma(\beta)} e^{-\lambda t} t^{\beta-1}, 0<\beta<1, \lambda>0
$$

[20] $\left(\right.$ then $\left.k(t)=\frac{1}{\Gamma(1-\beta)} e^{-\lambda t} t^{-\beta}+\frac{\lambda}{\Gamma(1-\beta)} \int_{0}^{t} e^{-\lambda \tau} \tau^{-\beta} d \tau\right)$;

$$
M(t)=\frac{1}{\Gamma(\beta)} e^{-\lambda t} t^{\beta-1}+\frac{\lambda}{\Gamma(\beta)} \int_{0}^{t} e^{-\lambda \tau} \tau^{\beta-1} d \tau, 0<\beta<1, \lambda>0
$$

[3] $\left(\right.$ then $\left.k(t)=\frac{1}{\Gamma(1-\beta)} e^{-\lambda t} t^{-\beta}\right)$;

$$
M(t)=e^{-\lambda t} t^{\beta-1} E_{\beta, \beta}\left(\lambda^{\beta} t^{\beta}\right), 0<\beta<1, \lambda>0,
$$

where $E_{\beta, \beta}$ is the two-parametric Mittag-Leffler function [22] (then $\left.k(t)=\frac{1}{\Gamma(1-\beta)} e^{-\lambda t} t^{-\beta}+\frac{\lambda}{\Gamma(1-\beta)} \int_{0}^{t} e^{-\lambda \tau} \tau^{-\beta} d \tau-\lambda^{\beta}\right)$.

\subsection{Positivity assumptions on $u_{x}$}

In this subsection we consider the direct problem (4)-(6), assume that its solution $u$ is sufficiently smooth and ask the question: which sufficient conditions on the data $f, h_{0}$ and $h_{l}$ guarantee the validity of the conditions $u_{x} \geq 0, D_{t}^{\{k\}} u_{x} \geq 0$ and $u_{x}(T, x)>0, x \in[0, l]$ in Theorems 3 and $4 ?^{1}$

\footnotetext{
1 Evidently, the other two inequality type conditions (28) and (29) in Theorem 3 follow from $u_{x}(T, x)>0, x \in[0, l]$.
} 
From (4)-(6) we deduce the following problem for $U=a u_{x}$ :

$$
\begin{aligned}
& D_{t}^{\{k\}} U(t, x)=a(x) U_{x x}(t, x)+a(x) f_{x}(t, x), x \in(0, l), t \in(0, T), \\
& U(t, 0)=h_{0}(t), U(t, l)=h_{l}(t), t \in(0, T), \\
& U(0, x)=0, x \in(0, l) .
\end{aligned}
$$

Assume that $a(x)>0, x \in[0, l]$. Then the conditions $u_{x} \geq 0, D_{t}^{\{k\}} u_{x} \geq 0$ and $u_{x}(T, x)>0, x \in[0, l]$ are equivalent to $U \geq 0, D_{t}^{\{k\}} U \geq 0$ and $U(T, x)>0, x \in[0, l]$, respectively. Moreover, let $k$ satisfy (12). Then we can apply a positivity principle for the generalized subdiffusion equation (Lemma 4 in [9]). The assumptions $f_{x} \geq 0, h_{0} \geq 0$ and $h_{l} \geq 0$ imply the inequality $U \geq 0$.

The problem for $V=D_{t}^{\{k\}} U$ reads

$$
\begin{aligned}
& D_{t}^{\{k\}}[V(t, x)-V(0, x)]=a(x) V_{x x}(t, x)+a(x) D_{t}^{\{k\}}\left[f_{x}(t, x)-f_{x}(0, x)\right], x \in(0, l), t \in(0, T), \\
& V(t, 0)=D_{t}^{\{k\}} h_{0}(t), V(t, l)=D_{t}^{\{k\}} h_{l}(t), t \in(0, T), \\
& V(0, x)=a(x) f_{x}(0, x), x \in(0, l) .
\end{aligned}
$$

Due to the above mentioned positivity principle, the assumptions $D_{t}^{\{k\}}\left[f_{x}(t, x)-f_{x}(0, x)\right] \geq 0, D_{t}^{\{k\}} h_{0} \geq 0$ and $D_{t}^{\{k\}} h_{l} \geq 0$ imply the inequality $D_{t}^{\{k\}} U \geq 0$.

It remains to deal with the strict inequality $U(T, x)>0, x \in[0, l]$. We are going to use a method presented in pp. 257-258 of [9]. Let us assume that

$$
\begin{aligned}
& \exists \mu \in C[0, T], \mu \geq 0, \mu \neq 0, \mu \text { - nondecreasing : } \\
& a(x) f_{x}(t, x) \geq \mu(t), x \in[0, l], t \in[0, T], h_{0}(t) \geq \mu(t), h_{l}(t) \geq \mu(t), t \in[0, T] .
\end{aligned}
$$

Let us denote $W=U-\delta 1 * \mu$ where $\delta>0$. The function $W$ is a solution to the problem

$$
\begin{aligned}
& D_{t}^{\{k\}} W(t, x)=a(x) W_{x x}(t, x)+a(x) f_{x}(t, x)-\delta k * \mu(t), x \in(0, l), t \in(0, T), \\
& W(t, 0)=h_{0}(t)-\delta 1 * \mu(t), W(t, l)=h_{l}(t)-\delta 1 * \mu(t), t \in(0, T), \\
& W(0, x)=0, x \in(0, l) .
\end{aligned}
$$

Let $\delta \leq \min \left\{\frac{1}{\int_{0}^{T} k(\tau) d \tau} ; \frac{1}{T}\right\}$. Then we have

$$
a(x) f_{x}(t, x)-\delta k * \mu(t) \geq a(x) f_{x}(t, x)-\delta \int_{0}^{t} k(\tau) d \tau \mu(t) \geq a(x) f_{x}(t, x)-\mu(t) \geq 0
$$

and $h_{0}(t)-\delta 1 * \mu(t) \geq h_{0}(t)-\delta t \mu(t) \geq h_{0}(t)-\mu(t) \geq 0$. Similarly, we obtain $h_{l}(t)-\delta 1 * \mu(t) \geq 0$. The positivity principle implies $W \geq 0$. Thus, $U(T, x)=W(T, x)+\delta 1 * \mu(T)>0, x \in[0, l]$.

\section{ACKNOWLEDGEMENTS}

The study was supported by the Estonian Research Council grant PRG832. The publications costs of this article were covered by the Estonian Academy of Sciences.

\section{REFERENCES}

1. Baeumer, B., Kurita, S. and Meerschaert, M. M. Inhomogeneous fractional diffusion equations. Fract. Calc. Appl. Anal., 2005, 8(4), 371-386. 
2. Chechkin, A. V., Gorenflo, R. and Sokolov, I. M. Fractional diffusion in inhomogeneous media. J. Phys. A: Math. Gen., 2005, 38(42), 679-684.

3. Gajda, J. and Magdziarz, M. Fractional Fokker-Planck equation with tempered $\alpha$-stable waiting times: Langevin picture and computer simulation. Phys. Rev. E., 2010, 82(1), 011117.

4. Gripenberg, G. On Volterra equations of the first kind. Integral Equ. Oper. Theory, 1980, 3(4), 473-488.

5. Gripenberg, G., Londen, S.-O. and Staffans, O. J. Volterra Integral and Functional Equations. Cambridge University Press, Cambridge, 1990.

6. Janno, J. and Kasemets, K. Uniqueness for an inverse problem for a semilinear time-fractional diffusion equation. Inverse Probl. Imaging, 2017, 11(1), 125-149.

7. Kian, Y., Oksanen, L., Soccorsi, E. and Yamamoto, M. Global uniqueness in an inverse problem for time fractional diffusion equations. J. Differ. Equ., 2018, 264(2), 1146-1170.

8. Kinash, N. and Janno, J. Inverse problems for a perturbed time fractional diffusion equation with final overdetermination. Math. Methods Appl. Sci., 2018, 41(5), 1925-1943.

9. Kinash, N. and Janno, J. Inverse problems for a generalized subdiffusion equation with final overdetermination. Math. Model. Anal., 2019, 24(2), 236-262.

10. Kinash, N. Inverse problems for generalized subdiffusion equations. PhD Thesis. Tallinn University of Technology, Estonia, 2020. https://digikogu.taltech.ee/et/Item/a3776907-fc6f-42e1-9d7c-5a0e6ae314f6

11. Kirane, M., Samet, B. and Torebek, B. T. Determination of an unknown source term temperature distribution for the subdiffusion equation at the initial and final data. Electron. J. Differ. Equ., 2017, 217, 1-13.

12. Mainardi, F., Mura, A., Pagnini, G. and Gorenflo, R. Time-fractional diffusion of distributed order. J. Vib. Control, 2008, 14(9-10), 1267-1290.

13. Metzler, R. and Klafter, J. The random walk's guide to anomalous diffusion: a fractional dynamics approach. Phys. Rep., 2000, 339(1), 1-77.

14. Orlovsky, D. Parameter determination in a differential equation of fractional order with Riemann - Liouville fractional derivative in a Hilbert space. J. Sib. Fed. Univ. Math. Phys., 2015, 8(1), 55-63.

15. Orlovsky, D. and Piskarev, S. Inverse problem with final overdetermination for time-fractional differential equation in a Banach space. J. Inverse Ill-Posed Probl., to appear.

16. Povstenko, Y. Z. Fractional heat conduction and associated thermal stress. J. Therm. Stresses, 2004, 28(1), 83-102.

17. Ren, C. and Xu, X. Local stability for an inverse coefficient problem of a fractional diffusion equation. Chin. Ann. Math. Ser. $B, 2014,35,429-446$.

18. Sakamoto, K. and Yamamoto, M. Inverse source problem with a final overdetermination for a fractional diffusion equation. Math. Control Relat. Fields, 2011, 1(4), 509-518.

19. Samko, S. G. and Cardoso, R. P. Integral equations of the first kind of Sonine type. Int. J. Math. Math. Sci., 2003, 2003, 238394.

20. Sandev, T., Metzler, R. and Chechkin, A. From continuous time random walks to the generalized diffusion equation. Fract. Calc. Appl. Anal., 2018, 21(1), 10-28.

21. Tuan, N. and Dinh, L. Fourier truncation method for an inverse source problem for space-time fractional diffusion equation. Electron. J. Differ. Equ., 2017, 2017(122), 1-16.

22. Wu, X., Deng, W. and Barkai, E. Tempered fractional Feynman-Kac equation: Theory and examples. Phys. Rev. E, 2016, 93(3), 032151.

23. Yamamoto, M. and Zhang, Y. Conditional stability in determining a zeroth-order coefficient in a half-order fractional diffusion equation by a Carleman estimate. Inverse Probl., 2012, 28(10), 105010.

\title{
Pöördülesanne difusioonikordaja määramiseks üldistatud subdifusioonivõrrandis lõpphetkel tehtud mõõtmiste alusel
}

\author{
Jaan Janno, Kairi Kasemets ja Nataliia Kinash
}

Vaadeldakse pöördülesannet difusioonikordaja määramiseks ühemõõtmelises subdifusioonivõrrandis, mis sisaldab üldistatud murrulist tuletist ajamuutuja suhtes. Ülesandes on lisatingimusena antud olekufunktsiooni jälg lõpphetkel $t=T$. On tõestatud pöördülesande lahendi ühesus. Seejärel on formuleeritud ligikaudsete algandmetega ülesanne ja tõestatud, et juhul, kui täpne ülesanne omab lahendit ja algandmete viga on piisavalt väike, siis omab ka ligikaudne ülesanne lahendit. Lisaks on tuletatud mainitud lahendite vahe hinnang algandmete vea kaudu. 